\title{
3 Research Square

\section{Transient Visual Disturbance is Associated with Disability and Suicidal Risk in Patients with Migraine Without Aura}

\section{Yu Chien Tsao}

Yonghe Cardinal Tien Hospital

\section{Yen-Feng Wang}

Taipei Veterans General Hospital

Jong-Ling Fuh

Taipei Veterans General Hospital

Wei-Ta Chen

Taipei Veterans General Hospital

Kuan-Lin Lai

Taipei Veterans General Hospital

Hung-Yu Liu

Taipei Veterans General Hospital

Shuu-Jiun Wang

Taipei Veterans General Hospital

Shih-Pin Chen ( $\nabla$ chensp1977@gmail.com )

Taipei Veterans General Hospital https://orcid.org/0000-0003-3492-9902

\section{Research article}

Keywords: Suicide risk, transient visual disturbance, photophobia

Posted Date: August 19th, 2021

DOI: https://doi.org/10.21203/rs.3.rs-762664/v1

License: (c) (1) This work is licensed under a Creative Commons Attribution 4.0 International License. Read Full License 


\section{Abstract}

Background: Patients with migraine without aura frequently report transient visual disturbances (TVD) other than typical visual aura, but the clinical correlates are not determined.

Method: Patients with migraine without aura (MO) that attended the headache clinic were enrolled. Structured questionnaires were self-administered by the patients including headache profiles, comorbidities, lifetime suicidal ideation and attempts, six-item Headache Impact Test, Migraine Photophobia Score, Migraine Disability Assessment, Hospital Anxiety and Depression Scale, Beck Depression Inventory, and Pittsburgh Sleep Quality Index. A semi-structured visual phenomenon questionnaire was also used to assess the characteristics of TVDs. TVD is defined as any zigzag flashes, flickering dots/lines, or blurred/foggy vision associated with headache and with a Visual Aura Rating Scale $<4$. Headache specialists interviewed with the participants for the ascertainment of diagnosis and verified of the questionnaires.

Result: MO patients ( $n=7,200$, female/male $=3.56$, mean age: $40.1 \pm 13.4$ years) were divided into 2 subgroups based on the presence $(n=2,488)$ or absence of TVDs $(n=4,712)$. Patients with TVD had higher headache-related disability, more psychiatric comorbidities and were more likely to be photophobic. Suicidal ideation and attempts were more common in patients with TVD than in those without (ideation: $31.9 \%$ vs. $18.1 \%, \mathrm{OR}=1.92$ [95\% Cl: $1.71-2.15], p<0.001$; attempt: $8.2 \%$ vs. $3.5 \%, \mathrm{OR}=2.23$ [95\% Cl: $1.80-2.75], p<0.001)$. The associations remained after adjustment of confounding factors.

Conclusion: Presence of transient visual disturbance may suggest a higher migraine-related disability, higher level of photophobia and higher suicidal ideation/attempts in MO patients.

\section{Introduction}

Migraine aura is reversible visual, sensory, or language disturbances associated with headache, which develops gradually over more than 5 minutes and lasts between 5 and 60 minutes [1,2]. Among these neurological symptoms, visual aura is the most common and occurs in $98 \%$ of patients with migraine with aura (MA) [3]. Flashes of bright light, foggy/blurred vision, zigzag/jagged lines are the most frequent visual aura symptoms [4]. In clinical practice, however, many patients with migraine without aura (MO) also reported different and complex transient visual disturbances (TVD) other than typical visual aura. Our previous field study in adolescents showed that almost half (48\%) of migraine adolescents without characteristic migraine visual aura experienced TVD related to their headache attack [5]. Moreover, there are some migraine patients predominantly affected by the visual symptoms due to variable duration or complexity, such as photophobia and after-image [6, 7]. Even though these TVDs do not fulfill the criteria of visual aura proposed by the International Classification of Headache Disorders (ICHD) [1, 2], they are frequently reported by patients with MO. However, they are poorly characterized, often neglected, and related research is limited. 
Previous studies have found connections between migraine aura and photophobia, which appear to be associated with cortical hypersensitivity [8]. Although the pathophysiology of TVD has not been fully investigated yet, we have found non-aura TVD is also associated with photophobia, implies the possibility of visual cortex hypersensitivity among patients with TVD [5]. On the other hand, studies have indicated that visual aura increased the odds of psychiatric comorbidities and suicide risk, possibly by alterations in the neuroendocrine system [9-11]. It is not known whether TVD could also be associated with psychiatric comorbidities and suicide risk among MO patients.

The aim of this study was to investigate the characteristics and clinical correlates of TVD among patients with migraine without visual aura, which may help understand the clinical significance of TVD and the complex neurobiology of migraine.

\section{Material And Methods}

\section{Study participants and data collection}

The headache specialists at the headache clinic of the Taipei Veterans General Hospital (TVGH) surveyed patients presented with headache during the period from May 2010 to July 2020. All participants completed a structured questionnaire to assess their headache profiles, comorbidities, mood, sleep, photophobic scale and suicidal ideation or attempts (see below) at their first visit. A semi-structured visual phenomenon questionnaire was used to assess the characteristics of TVDs. Later, they underwent a thorough clinical interview for the ascertainment of headache diagnosis as well as the questionnaires. Information collected from the questionnaires was de-identified and entered into the TVGH headache registry.

\section{Ethics}

This retrospective study analyzed these de-identified data included in the TVGH headache registry. Because this study involved secondary analysis of existing de-identified data, informed consent has been waived by the institutional review board of Taipei Veterans General Hospital, which approved the whole study protocol (TVGH IRB-2021-04-121-CC). The corresponding authors had full access to all of the data in the study and had final responsibility for the decision to submit for publication.

\section{Diagnoses of migraine}

The diagnoses of MA, MO, and chronic migraine (CM) were based on the International Classification of Headache Disorders, 2nd and 3rd edition (ICHD-2 and ICHD-3) criteria (MO: code 1.1; MA: code 1.2; CM: code 1.3) [1, 2], made through face-to-face interviews by experienced headache specialists. Patients with MA were excluded from this study.

\section{Definition of TVD}

TVDs were defined as transient visual phenomena related in time to the occurrence of a migraine headache attack, but not visual aura. All of the participants received the five items of the Visual Aura 
Rating Scale (VARS) embedded in the questionnaires. The score is the weighted sum of the five-item scale: duration 5-60 minutes (three points), developing gradually $\geq 5$ minutes (two points), scotoma (two points), zig-zag lines (two points) and unilateral visual field (one point). A cutoff VARS score of five or more points is reportedly highly sensitive and specific to diagnose MA [12]. In order to exclude potential aura, we also explored the VARS cutoff value in our cohort. MA diagnoses made by neurologists based on ICHD [1, 2] were used as the gold standard. Compared to physician diagnosis, a VARS score $\geq$ five had a sensitivity of $68.4 \%$ and a specificity of $88.7 \%$, while a VARS score $\geq 4$ had a sensitivity of $77.1 \%$ and a specificity of $79.9 \%$ to identify typical migraine aura. By using the criteria, i.e. a VARS score of $\geq 4$, the positive predictive value (PPV) was 0.29 and the negative predictive value (NPV) was 0.97 for MA. Hence, in this study, we included only MO patients (diagnosed by neurologists) with VARS $<4$ to ensure that their TVDs are unlikely to be visual aura.

\section{Questionnaires}

Demographic data, including age, sex, occupation, education level, marital status, and medical history were collected. A validated headache questionnaire was used to specifically inquire headache frequency (days/ month), intensity (Numerical Rating Scale 0-10), duration, location, characteristics, accompanying symptoms, frequency of acute abortive medications usage (days/month), and disease duration of migraine (years), as well as TVD symptoms $[13,14]$. To differentiate these visual symptoms from characteristic migraine aura defined in the ICHD, a visual phenomenon questionnaire was used to assess the TVDs, including patterns (zigzag flashes, flickering dots/lines, or blurred/foggy vision), laterality of the visual fields, colors, presence of movement, development time, duration and temporal relationship with headaches. The questions were:

1. Have you ever seen zigzag flashes before or during the headache?

2. Have you ever seen flickering dots or lines before or during the headache?

3. Before or when the headache started, did you have blurred or foggy vision?

4. What colors are these zigzag flashes, flickering dots or lines?

5. Do these TVDs (zigzag flashes, flickering dots or lines, or blurred vision) occur every time you have a headache?

6. Are these TVDs that you have seen unilateral, bilateral or different every time?

7. Did your TVDs move?

8. How long did your TVDs develop?

9. How long did your TVDs last?

10. Did these TVDs develop before, after or during the onset of the headache?

Afterwards, the MO patients were divided in to 2 subgroups based on the presence (MwTVD) or absence (MwoTVD) of TVDs. Additionally, the questionnaires also include the Migraine Disability Assessment (MIDAS), six-item Headache Impact Test (HIT-6), Migraine Photophobia Score (MPS), Hospital Anxiety and Depression Scale (HADS), Beck Depression Inventory (BDI), Perceived Stress Scale (PSS), and 
Pittsburgh Sleep Quality Index (PSQI), presence of $\mathrm{MOH}$, suicidal ideation and attempts to collect relevant clinical information.

The Migraine Disability Assessment (MIDAS) questionnaire assesses headache-related disability in a 3month period and the six-item Headache Impact Test (HIT-6) measures the severity of headache pain and adverse impact of headache $[15,16]$. Both MIDAS and HIT-6 have been well accepted and widely utilized to evaluate the disability and impact caused by migraine. Headache intensity influences HIT-6 score more than the MIDAS, whereas the MIDAS is influenced more by headache frequency [17]. Migraine Photophobia Score (MPS) is a self-administered, eight-question questionnaires to evaluate the scale of photophobia in migraine patients. Adding the total number of "yes" responses generates the MPS [18]. Hospital Anxiety and Depression Scale (HADS) is a self-administered instrument to detect psychiatric comorbidity in the setting of a hospital outpatient clinic [19]. Anxiety was defined as a HADS score $\geq 13$ based on our previous study[20]. The Beck Depression Inventory (BDI) is a 21-item self-report measure that evaluates major depression symptoms according to diagnostic criteria listed in the Diagnostic and Statistical Manual for Mental Disorders [21], and moderate depression is defined as a BDI $\geq 20$ [22, 23]. The Perceived Stress Scale (PSS) is a 14-item self-reported questionnaire that was designed to measure "the degree to which individuals appraise situations in their lives as stressful" [24, 25]. Pittsburgh Sleep Quality Index (PSQI) evaluates the quality and patterns of sleep in the past one month. Poor sleep quality was defined as a PSQI score of $>5$ [26]. Suicidal ideation and attempts were evaluated by 2 separate directly asked questions: (1) whether they, once in their lifetime, had ideational thoughts of engaging in suicidal behavior, and (2) whether they had engaged in any self-injury behavior with the intent to die. Unanswered questionnaires were interpreted as missing data.

\section{Migrainous features, the prevalence of visual disturbance, and the severity of photophobia}

Migrainous features (including moderate to severe intensity; pulsating quality; unilaterality; aggravation by physical activity; nausea or vomiting; photophobia and photophobia) of each subject were evaluated. The "yes" responses to each features were summed up to a total score of migrainous features, ranging from 0 to 6 . To evaluate the migrainous feature in patients with photophobia, we re-calculated numbers of migrainous features ranged from 0 to 5 , including the same features mentioned above except photophobia and phonophobia.

\section{Statistical analysis}

The descriptive data were presented as means \pm SDs or percentages. The Chi square test was used to test the difference in categorical data. Continuous data between groups were analyzed using Student's t test with Bonferroni correction. For subgroup analysis, logistic regression was performed to test the interaction effect. The risks associated with comorbid suicidal ideation and attempts were predicted separately by 3 layers of models: (1) no controlling for any covariates; (2) controlling for demographics; (3) controlling for demographics and clinical characteristics. These models were performed stepwise. Demographics included patients' age, sex, and marital status (married vs. 
single/separated/divorced/widowed). Clinical characteristics included headache frequency (days/month), HIT-6 score, depression (BDI $\geq 20$ ), anxiety (HADS score $\geq 13$ ), poor sleep quality (PSQI > 5), $\mathrm{MOH}$, and $\mathrm{CM}$. The predictors were presented as odds ratio (OR) with $95 \%$ confidence interval. Results were considered significant for $p$ value $<0.05$. Statistical analyses were performed with $\mathrm{R}$ for Mac OS (Version 3.6.3; R Core Team, Vienna, Austria.)

\section{Results}

\section{Prevalence of TVD}

Totally 12,255 patients who visited our headache clinic during the 10-year study period were enrolled. Migraine was diagnosed in 9,966 patients, while the other 2,309 patients were excluded due to nonmigraine headache. Among the migraineurs, 962 patients diagnosed with MA and 1,784 MO patients with VARS $\geq 4$ were excluded from the study. After excluding those with aura or high VARS, the remaining 7,200 patients with MO constituted the final sample. The MO patients (female/male 5,620 (78.1\%)/1,580 (21.9\%); mean age: $40.1 \pm 13.4$ years) were divided into 2 subgroups based on the presence (MwTVD, $n=$ $2,488,34.6 \%$ ) or absence (MwoTVD, $n=4,712,65.4 \%$ ) of TVD. A flow chart of the patient enrollment process is presented in Figure 1.

\section{TVD characteristics and clinical features}

Among the 2,488 MwTVD subjects, 865 (34.8\%) patients reported more than one pattern of TVDs. The most common TVD was blurred or foggy vision $(n=1,766,71.0 \%)$, followed by flickering dots/lines $(n=$ $1,332,53.5 \%)$ and zigzag flashes $(n=279,11.2 \%)$. More than a quarter of the patients $(n=683,27.5 \%)$ reported both positive TVDs (flickering dots /lines, or zigzag flashes) and negative TVDs (blurred/foggy vision). Most of the TVDs happened during the headache (74.1\%), while 18.4\% happened before headache and $7.5 \%$ after the headache. The onset of TVD was commonly quick (59.2\% of VD occurred in $\leq 30$ seconds, $22.7 \%$ between 30 seconds to 1 minute, and $15.3 \%$ between $1-5$ minutes), and the duration was also short ( $51.6 \%$ lasted $\leq 30$ seconds, $23.0 \%$ lasted for 30 seconds to 1 minute, and $15.8 \%$ lasted for 1-5 minutes). The detailed characteristics of the TVDs are shown in Table 1. The onset time, duration, and temporal relationships with headache are the three major different characteristics of TVD comparing with typical visual aura. To validate phenotype-based diagnosis of TVD, we compared these three characteristics between our MWTVD and MA groups. For visual symptoms that did not fulfill typical visual aura but had at least two of these three characteristics: A. Develops or spreads in less than 5 minutes; $\mathrm{B}$. Lasts last than 5 minutes; $\mathrm{C}$. Occurs during headache phase, the sensitivity and specificity to identify TVD were $96.38 \%$ and $64.03 \%$. Both the PPV and NPV were 0.87 .

Details of demographic data and clinical characteristics of MwTVD and MwoTVD are listed in Table 2. In general, MwTVD had worse clinical features than MwoTVD. The MwTVD group, compared with the MwoTVD group, had higher headache frequency, more severe headache-related disability, higher proportions of $\mathrm{CM}, \mathrm{MOH}$ and psychiatric comorbidities, and were more likely to be photophobic. 
There is a trend for increasing TVD frequency with increasing migrainous features (Fig. 2A). While the TVD was reported in only $12.2 \%$ of $\mathrm{MO}$ patients with least migrainous features, its prevalence increased to $46.6 \%$ in those with all six migrainous features. To investigate whether patients with TVD were also more photophobic, we further compared the MPS between MwTVD and MwoTVD. Consistent with our speculation, MwTVD patients had higher MPS while comparing with MwoTVD (2.9 \pm 2.0 vs. $1.6 \pm 1.9, p<$ 0.001 , Table 1). Moreover, we found MPS was a predictor of TVD (unadjusted OR $=1.37,95 \% \mathrm{Cl}=1.32-$ $1.42, p<0.001$ ), and the result remained after gender and age were adjusted (adjusted $\mathrm{OR}=1.36,95 \% \mathrm{Cl}$ $=1.31-1.41, p<0.001)$. Similar to TVD, there is also a trend for increasing photophobia frequency with increasing migrainous features, expanding from $15.6 \%$ to $38.4 \%$ (Fig. 2B).

\section{Suicide risk in patients with migraine with visual disturbance and photophobia}

Because the percentages of suicidal ideation and attempts were much higher in the MwTVD group than those in the MwoTVD group (31.9\% vs. $18.1 \%, p<0.001$ for suicidal ideation; $8.2 \%$ vs. $3.5 \%, p<0.001$ for suicide attempts, Table 2), we further explored whether TVD could be an independent risk factor for suicidal risk in patients with migraine without typical visual aura. Univariate analysis showed that TVD, photophobia, headache frequency and headacherelated disability, $\mathrm{CM}, \mathrm{MOH}$, along with traditional risk factors including depression, anxiety, and poor sleep quality, were associated with higher risks of suicidal ideation and attempts in patients with migraine, while marriage had a protective effect in suicide ideation (Table 3). In multivariable analysis, TVD remained an independent risk factor for suicidal ideation and attempts even after controlling for demographics, headache frequency, headache-related disability, CM, $\mathrm{MOH}$ and psychiatric comorbidities (Table 4).

\section{Discussion}

Our study demonstrated that the prevalence of the non-aura TVD was as high as $34.6 \%$ among MO patients. Patients with TVD are predominately female, had worse headache-related disability, more psychiatric comorbidities, and were more likely to be photophobic compared with patients without TVD. Patients who exhibited more migrainous features were more likely to have a higher prevalence of TVD and photophobia, implying the clinical significance of non-aura visual symptoms in migraine. Moreover, the presence of TVD was positively correlated with increased suicidal ideation and attempts, even after the other suicide risk factors were adjusted. Based on these findings, we speculated that the presence of TVD might serve as a marker of disease severity and even a potential indicator of higher suicidal risk.

Blurred/foggy vision is the most common type of TVD, and more than a quarter of these patients (27.5\%) also have seen flickers or zigzag flashes. This is similar to a previous study, stating that "blurred/foggy vision" was the most common visual symptom other than aura among MA patients [27]. The TVDs reported in our patients are mostly fixed, colorless, over non-specific fields, developing within 5 minutes, lasting less than 5 minutes, and occurring during the headache phase. This finding is consistent with our previous study indicating the transient TVDs are short in onset to development as well as the duration 
and is common among adolescent patients with migraine [5]. The major differences between typical visual aura and TVD are the onset and duration of the symptoms, and the temporal relation with headache. Also, among our 9,946 migraine patients, one-fourth of them reported to have non-aura TVD $(n=2,488)$ but only less than one-tenth of then have MA $(n=962)$, showing that the prevalence of TVD is much higher than that of typical aura. We proposed that these non-classic visual symptoms should not be classified as atypical aura, and might be identified separately. We suggest non-aura TVD has crucial clinical implications and should be identified in clinical practice. Herein we proposed an operational criteria to characterize these migraine-associated TVD, the clinical utility of which needs be validated in future studies (Table 5).

To our best knowledge, this is the first study that found TVD is related to higher migraine-related disability, higher level of photophobia, and higher suicidal ideation/attempts risk. Furthermore, the odds ratios of TVD and MPS on suicide are higher than HIT- 6 and headache frequency, which were previously thought to be highly related to suicide risk [28]. This implies the impact of non-aura TVD on migraine might be underestimated. Also, both TVD and photophobia have a higher prevalence among those with more migrainous features than those without (Fig. 2). Although photophobia is known as one of the most disabling symptoms of migraine [29], the disabilities caused by TVD are seldom addressed. Therefore, we propose that TVD should be considered as one of the indicators to evaluate the prognosis of migraine.

The pathophysiology of TVD in patients with migraine is unknown. Typical aura is associated with abnormal cortical hyper-excitability, and visual percept of aura could change depends on the region of the occipital cortex involved [30]. Previous studies also found cortical hypersensitivity might be the link between migraine aura and photophobia [8]. Since patients with TVD were more photophobic than those without and that increased interictal visual sensitivity is present in both MA and MO [31], the TVD in some patients might also be linked to mechanisms similar to cortical hyper-excitability but involving a less eloquent visual cortex [32]. We speculate that our patients with TVD may have a hyperexcitable visual cortex, which contributes to non-aura TVD and more severe photophobia and may thus lead to worse migraine-related disability. Furthermore, we found non-aura TVD as an independent risk factor of increased suicidal ideation or attempts even after adjusting other potential confounders. Studies have reported that suicide survivors had lower oxytocin concentration both in serum and CSF [33,34], and oxytocin is a potential biomarker of attempted suicide [35]. On the other hand, oxytocin released from paraventricular neurons (PVN) can suppress nociception of inflammatory pain [36]. Since PVN also channels the photic input from the retina [37], it is possible that oxytocin may be also related to migrainetype photophobia and worse headache-related disability. However, it is hypothetical and systematic studies should be conducted in the future.

There are several strengths in our study. First, our sample size is large, with more than 7,000 patients with $\mathrm{MO}$ enrolled consecutively in the ten-year study period. The large sample size increased the validity of this clinic-based study. Second, we have detailed and validated questionnaires to specifically inquire headache characteristics and TVD symptoms. The data from the questionnaires was highly qualified, and these questionnaires have been widely accepted as reliable neuropsychological instruments in many 
studies $[13,14,20]$. Furthermore, the final diagnoses of $M O$ and TVD were ascertained by experienced headache specialists made through face-to-face interview, which help to confirm the accuracy of our data. We also used VARS to exclude the potential inclusion of aura. Additionally, we provided our proposed TVD diagnostic criteria based the phenotype of the visual symptoms. Therefore, we believe our findings could have a practical implication for neurologists.

However, there are also some limitations in our study. First, all the patients were recruited from a tertiary medical center; thus, the patients we seen were at the worse end of the disease spectrum. Nevertheless, even in these patients, those with TVD still had much worse clinical features than those without. Second, the suicide risk was evaluated based on single-item direct questions, questioning of the suicidal ideation and attempts. Although the patients received face-to-face interview, detailed information regarding the potential etiologies of suicidal ideation or attempts was not systematically collected. However, the overall lifetime suicidal attempts in our MwoTVD was very close to previous cross-national, multicultural study (3.5\% and $2.7 \%$, respectively), indicating the reliability of our study [38]. As previous studies have shown that more than half of suicidal ideations transform into plan and attempt in one year, and suicide attempt is the best predictor of a completed suicide [39]. Last, we focused on psychiatric diseases and some other demographics factors since these are the strongest predictors of suicide while some other risk factors for suicide such as chronic pain disorders, fibromyalgia, major life events, and financial status were not controlled in the multivariable analysis [40,41]. Further study to investigate these risk factors should be conducted in the future.

\section{Conclusions}

In this study, we found that non-aura TVD was associated with higher level of photophobia, higher migraine-related disability, and higher suicidal ideation/attempts. The odds ratio of TVD and MPS on suicide are higher than HIT- 6 and headache frequency, implies the impact of non-aura TVD and photophobia on migraine might be underestimated. We suggested that TVD should be considered as one of the indicators to evaluate the prognosis of migraine, and proposed an operational criteria for TVD. Nevertheless, the exact pathophysiology is unknown and required further exploration.

\section{Abbreviations}

BDI: Beck Depression Inventory; CDH: Chronic daily headache; Cl: Confidence interval; CM: Chronic migraine; HADS: Hospital Anxiety and Depression Scale; HAS: Hospital Anxiety Scale; HDS: Hospital Depression Scale; HIT-6: six-item Headache Impact Test; ICHD: The International Classification of Headache Disorders; MIDAS: Migraine Disability Assessment Scale; MOH: Medication-overuse headache; MPS: Migraine Photophobia Score; OR: Odds ratio; PSQI: Pittsburgh Sleep Quality Index; PSS: Perceived Stress Scale; TVD: Transient visual disturbance.

\section{Declarations}




\section{Ethics approval and consent to participate}

This retrospective study analyzed these de-identified data included in the TVGH headache registry.

Because this study involved secondary analysis of existing de-identified data, informed consent has been waived by the institutional review board of Taipei Veterans General Hospital, which approved the whole study protocol (TVGH IRB-2021-04-121-CC). The corresponding authors had full access to all of the data in the study and had final responsibility for the decision to submit for publication.

\section{Consent for publication}

Not applicable.

\section{Availability of data and materials}

The datasets used and/or analyzed during the current study are available from the corresponding author on reasonable request.

\section{Competing interests}

The authors have declared no relevant conflict of interests.

\section{Funding}

This work was supported by the Brain Research Center, National Yang Ming Chiao Tung University from The Featured Areas Research Center Program within the framework of the Higher Education Sprout Project by the Ministry of Education (MOE) in Taiwan (to SJW \& SPC); the Ministry of Science and Technology, Taiwan [MOST-107-2314-B-010-021, 108-2314-B-010 -022 -MY3 \& 110-2326-B-A49A-501 MY3 (to SPC) and MOST 108-2321-B-010-014-MY2, 108-2321-B-010-001-, 108-2314-B-010-023-MY3 \& 110-2321-B-010-005- (to SJW) ], Ministry of Health and Welfare, Taiwan [MOHW107-TDU-B-211-123001 and MOHW 108-TDU-B-211-133001] (to SJW), and Taipei Veterans General Hospital, Taiwan [VGH-106D9-001-MY2-2 (to SJW) \& V110C-102, V109D52-001-MY3-2, VGHUST110-G1-3-1 (to SPC)]. The funders had no role in study design, data collection and analysis, decision to publish, or preparation of the manuscript.

\section{Authors' contributions}

YCT and SPC conceived and designed the study, and completed the first draft. JLF, YFW, WTC, KLL, HYL and SJW acquired and analyzed the data. SPC and SJW participated in the discussion and critical revision of the manuscript. The authors have read and approved the manuscript

\section{Acknowledgements}

The authors would like to thank the study participants for their contributions.

\section{Conflicts of interest:}


The authors declare that they have no conflicts of interest related to the subject matter or materials discussed in this article.

\section{References}

1. Headache Classification Subcommittee of the International Headache Society (2004) The International Classification of Headache Disorders: 2nd edition. Cephalalgia 24 Suppl 1:9-160.

2. Headache Classification Committee of the International Headache Society (IHS) (2018) The International Classification of Headache Disorders, 3rd edition. Cephalalgia 38(1):1-211.

3. Viana M, Sances G, Linde M, Ghiotto N, Guaschino E, Allena M et al (2017) Clinical features of migraine aura: results from a prospective diary-aided study. Cephalalgia 37(10):979-989.

4. Viana M, Tronvik EA, Do TP, Zecca C, Hougaard A (2019) Clinical features of visual migraine aura: a systematic review. J Headache Pain 20(1):64.

5. Liu HY, Fuh JL, Lu SR, Chen SP, Chou CH, Wang YF et al (2012) Transient visual disturbances in adolescents: migrainous feature or headache-accompanied phenomenon? Cephalalgia 32(15):11091115.

6. van Dongen RM, Haan J (2019) Symptoms related to the visual system in migraine. F1000Res 8.

7. Luedtke K, Schulte LH, May A (2019) Visual processing in migraineurs depends on the migraine cycle. Ann Neurol 85(2):280-283.

8. Hayne DP, Martin PR (2019) Relating Photophobia, Visual Aura, and Visual Triggers of Headache and Migraine. Headache 59(3):430-442.

9. Purselle DC, Nemeroff CB (2003) Serotonin transporter: a potential substrate in the biology of suicide. Neuropsychopharmacology 28(4):613-619.

10. Wang SJ, Fuh JL, Juang KD, Lu SR (2009) Migraine and suicidal ideation in adolescents aged 13 to 15 years. Neurology 72(13):1146-1152.

11. Radat F, Swendsen J (2005) Psychiatric comorbidity in migraine: a review. Cephalalgia 25(3):165178.

12. Eriksen MK, Thomsen LL, Olesen J (2005) The Visual Aura Rating Scale (VARS) for migraine aura diagnosis. Cephalalgia 25(10):801-810.

13. Wang YF, Yu CC, Kuan AS, Chen SP, Wang SJ (2021) Association between suicidal risks and medication-overuse headache in chronic migraine: a cross-sectional study. $J$ Headache Pain 22(1):36.

14. Hung $\mathrm{Cl}$, Liu CY, Yang CH, Wang SJ (2015) The Impacts of Migraine among Outpatients with Major Depressive Disorder at a Two-Year Follow-Up. PLoS One 10(5):e0128087.

15. Stewart WF, Lipton RB, Whyte J, Dowson A, Kolodner K, Liberman JN et al (1999) An international study to assess reliability of the Migraine Disability Assessment (MIDAS) score. Neurology 53(5):988-994. 
16. Kosinski M, Bayliss MS, Bjorner JB, Ware JE, Jr., Garber WH, Batenhorst A et al (2003) A six-item short-form survey for measuring headache impact: the HIT-6. Qual Life Res 12(8):963-974.

17. Sauro KM, Rose MS, Becker WJ, Christie SN, Giammarco R, Mackie GF et al (2010) HIT-6 and MIDAS as measures of headache disability in a headache referral population. Headache 50(3):383-395.

18. Choi JY, Oh K, Kim BJ, Chung CS, Koh SB, Park KW (2009) Usefulness of a photophobia questionnaire in patients with migraine. Cephalalgia 29(9):953-959.

19. Zigmond AS, Snaith RP (1983) The hospital anxiety and depression scale. Acta Psychiatr Scand 67(6):361-370.

20. Juang KD, Wang SJ, Lin CH, Fuh JL (1999) Use of the hospital anxiety and depression scale as a screening tool for patients with headache. Zhonghua Yi Xue Za Zhi (Taipei) 62(11):749-755.

21. American Psychiatric Association (2013) Diagnostic and statistical manual of mental disorders (DSM-5®)

22. Beck AT, Ward CH, Mendelson M, Mock J, Erbaugh J (1961) An inventory for measuring depression. Arch Gen Psychiatry 4:561-571.

23. Wang YP, Gorenstein C (2013) Psychometric properties of the Beck Depression Inventory-II: a comprehensive review. Braz J Psychiatry 35(4):416-431.

24. Lee EH (2012) Review of the psychometric evidence of the perceived stress scale. Asian Nurs Res (Korean Soc Nurs Sci) 6(4):121-127.

25. Cohen S (1988) Perceived stress in a probability sample of the United States.

26. Buysse DJ, Reynolds CF, 3rd, Monk TH, Berman SR, Kupfer DJ (1989) The Pittsburgh Sleep Quality Index: a new instrument for psychiatric practice and research. Psychiatry Res 28(2):193-213.

27. Queiroz LP, Rapoport AM, Weeks RE, Sheftell FD, Siegel SE, Baskin SM (1997) Characteristics of migraine visual aura. Headache 37(3):137-141.

28. Lin YK, Liang CS, Lee JT, Lee MS, Chu HT, Tsai CL et al (2019) Association of Suicide Risk With Headache Frequency Among Migraine Patients With and Without Aura. Front Neurol 10:228.

29. Walters AB, Smitherman TA (2016) Development and Validation of a Four-Item Migraine Screening Algorithm Among a Nonclinical Sample: The Migraine-4. Headache 56(1):86-94.

30. Hansen JM, Baca SM, Vanvalkenburgh P, Charles A (2013) Distinctive anatomical and physiological features of migraine aura revealed by 18 years of recording. Brain 136(Pt 12):3589-3595.

31. Cucchiara B, Datta R, Aguirre GK, Idoko KE, Detre J (2015) Measurement of visual sensitivity in migraine: Validation of two scales and correlation with visual cortex activation. Cephalalgia 35(7):585-592.

32. Schankin CJ, Viana M, Goadsby PJ (2017) Persistent and Repetitive Visual Disturbances in Migraine: A Review. Headache 57(1):1-16.

33. Jahangard L, Shayganfard M, Ghiasi F, Salehi I, Haghighi M, Ahmadpanah M et al (2020) Serum oxytocin concentrations in current and recent suicide survivors are lower than in healthy controls. Journal of Psychiatric Research 128:75-82. 
34. Jokinen J, Chatzittofis A, Hellström C, Nordström P, Uvnäs-Moberg K, Asberg M (2012) Low CSF oxytocin reflects high intent in suicide attempters. Psychoneuroendocrinology 37(4):482-490.

35. Parris MS, Grunebaum MF, Galfalvy HC, Andronikashvili A, Burke AK, Yin H et al (2018) Attempted suicide and oxytocin-related gene polymorphisms. J Affect Disord 238:62-68.

36. Nersesyan Y, Demirkhanyan L, Cabezas-Bratesco D, Oakes V, Kusuda R, Dawson T et al (2017) Oxytocin Modulates Nociception as an Agonist of Pain-Sensing TRPV1. Cell Rep 21(6):1681-1691.

37. Mikkelsen JD, Larsen PJ, Mick G, Vrang N, Ebling FJ, Maywood ES et al (1995) Gating of retinal inputs through the suprachiasmatic nucleus: role of excitatory neurotransmission. Neurochem Int 27(3):263-272.

38. Nock MK, Borges G, Bromet EJ, Alonso J, Angermeyer M, Beautrais A et al (2008) Cross-national prevalence and risk factors for suicidal ideation, plans and attempts. Br J Psychiatry 192(2):98-105.

39. Mościcki EK (1997) Identification of suicide risk factors using epidemiologic studies. Psychiatr Clin North Am 20(3):499-517.

40. Yuodelis-Flores C, Ries RK (2015) Addiction and suicide: A review. Am J Addict 24(2):98-104.

41. Liu HY, Fuh JL, Lin YY, Chen WT, Wang SJ (2015) Suicide risk in patients with migraine and comorbid fibromyalgia. Neurology 85(12):1017-1023.

\section{Tables}

Table 1. Characteristics of TVDs 


\begin{tabular}{|c|c|}
\hline & $n(\%)^{a}$ \\
\hline Blurred /foggy vision & $1,766(71.0 \%)$ \\
\hline Flickering dots or lines & $1,332(53.5 \%)$ \\
\hline Zigzag flashes & $279(11.2 \%)$ \\
\hline Hazy & $227(47.7 \%)$ \\
\hline Wave-like & $153(32.1 \%)$ \\
\hline Fortification spectra & $58(12.2 \%)$ \\
\hline Reticular & $38(8.0 \%)$ \\
\hline \multicolumn{2}{|l|}{ Colors } \\
\hline Colorless & $653(38.6 \%)$ \\
\hline Whitish & $641(37.9 \%)$ \\
\hline Blackish & $184(10.9 \%)$ \\
\hline Non-specific & $94(5.6 \%)$ \\
\hline Yellowish & $83(4.9 \%)$ \\
\hline Rainbow like & $36(2.1 \%)$ \\
\hline \multicolumn{2}{|c|}{ Co-occurrence of VD and headache } \\
\hline Not every time & $2,167(92.7 \%)$ \\
\hline Every time & $170(7.3 \%)$ \\
\hline \multicolumn{2}{|l|}{ Laterality } \\
\hline Non-specific & $908(36.4 \%)$ \\
\hline Bilateral & $688(27.7 \%)$ \\
\hline Unilateral & $892(35.9 \%)$ \\
\hline \multicolumn{2}{|l|}{ Movable } \\
\hline Yes & $921(40.4 \%)$ \\
\hline No & $1,356(59.6 \%)$ \\
\hline \multicolumn{2}{|l|}{ Time of development } \\
\hline$\leq 30$ seconds & $1,472(59.2 \%)$ \\
\hline 0.5 - 1 minute & $564(22.7 \%)$ \\
\hline 1 - 5 minutes & $380(15.3 \%)$ \\
\hline
\end{tabular}




\begin{tabular}{ll}
$\mathbf{5}$ - 20 minutes & $36(1.4 \%)$ \\
\hline$\geq 20$ minutes & $36(1.4 \%)$
\end{tabular}

Duration
\begin{tabular}{ll}
$\mathbf{5} \mathbf{3 0}$ seconds & $1,285(51.6 \%)$ \\
\hline $\mathbf{0 . 5}-\mathbf{1}$ minute & $571(23.0 \%)$ \\
\hline $\mathbf{1}-\mathbf{5}$ minutes & $393(15.8 \%)$ \\
\hline $\mathbf{5}-\mathbf{3 0}$ minutes & $20(0.8 \%)$ \\
\hline $\mathbf{0 . 5}-\mathbf{1}$ hour & $13(0.5 \%)$ \\
$\mathbf{2} \mathbf{1}$ hour & $206(8.3 \%)$ \\
\hline
\end{tabular}

Temporal relationship with headache

\begin{tabular}{ll} 
Before headache & $397(18.4 \%)$ \\
\hline During headache & $1,602(74.1 \%)$ \\
\hline After headache & $162(7.5 \%)$ \\
\hline
\end{tabular}

aThe data represent the number of subjects who answered each questionnaire; unanswered questionnaires were interpreted as missing data; TVD: transient visual disturbance.

Table 2. Demographic data, headache characteristics and comorbidity in patients with migraine with (MwTVD) or without transient visual disturbance (MwoTVD) 


\begin{tabular}{|c|c|c|c|}
\hline & $\begin{array}{l}\text { MwTVD } \\
n=2,488\end{array}$ & $\begin{array}{l}\text { MwoTVD } \\
n=4,712\end{array}$ & $p$ value \\
\hline Age & $38.2 \pm 13.0$ & $41.1 \pm 13.5$ & $<2 \mathrm{e}-16^{\star}$ \\
\hline Gender (Female/Male); n (\%) & $\begin{array}{l}2,023(81.3) / 465 \\
(18.7)\end{array}$ & $\begin{array}{l}3,597(76.3) / 1,115 \\
(23.7)\end{array}$ & $1.242 \mathrm{e}-06^{*}$ \\
\hline Headache days/ month & $13.0 \pm 9.6$ & $10.7 \pm 9.3$ & $<2.2 \mathrm{e}-16^{\star}$ \\
\hline MPS & $2.9 \pm 2.0$ & $1.6 \pm 1.9$ & $<2.2 \mathrm{e}-16^{\star}$ \\
\hline MIDAS & $31.8 \pm 44.9$ & $22.9 \pm 35.9$ & $<2.2 \mathrm{e}-16^{\star}$ \\
\hline HIT-6 & $62.6 \pm 6.6$ & $60.7 \pm 7.8$ & $2.105 \mathrm{e}-14^{*}$ \\
\hline HADS & $15.3 \pm 7.6$ & $12.30 \pm 7.2$ & $<2.2 \mathrm{e}-16^{\star}$ \\
\hline HAS & $8.8 \pm 4.3$ & $7.1 \pm 4.1$ & $<2.2 \mathrm{e}-16^{\star}$ \\
\hline HDS & $6.5 \pm 4.1$ & $5.3 \pm 3.9$ & $<2.2 \mathrm{e}-16^{\star}$ \\
\hline BDI & $13.1 \pm 9.1$ & $9.7 \pm 7.5$ & $<2.2 \mathrm{e}-16^{\star}$ \\
\hline PSS & $26.5 \pm 9.2$ & $23.8 \pm 8.6$ & $<2.2 \mathrm{e}-16^{\star}$ \\
\hline PSQI & $10.1 \pm 4.2$ & $8.6 \pm 4.0$ & $2.734 \mathrm{e}-15^{\star}$ \\
\hline Chronic migraine & $902(36.3 \%)$ & $1,327(28.2 \%)$ & $1.634 \mathrm{e}-12^{\star}$ \\
\hline $\begin{array}{l}\text { Medication overuse } \\
\text { headache }\end{array}$ & $469(18.9 \%)$ & $806(17.1 \%)$ & 0.06507 \\
\hline Suicidal ideation & $793(31.9 \%)$ & $854(18.1 \%)$ & $4.437 \mathrm{e}-30 *$ \\
\hline Suicide attempt & $203(8.2 \%)$ & $167(3.5 \%)$ & $2.969 \mathrm{e}-14^{*}$ \\
\hline
\end{tabular}

Abbreviations: MIDAS, Migraine Disability Assessment; HIT-6, six-item Headache Impact Test; MPS, Migraine Photophobia Score; HDAS, Hospital Anxiety and Depression Scale; HDS, Hospital depression scale; HAS, Hospital Anxiety Scale; BDI, Beck Depression Inventory; PSS, Perceived Stress Scale; PSQI, Pittsburgh Sleep Quality Index. Data are presented as mean \pm SD. * Significant after Bonferroni correction

Table 3. Association of suicide ideation/attempts with potential risk factors. 


\begin{tabular}{|lllll|}
\hline & Suicidal ideation & & Suicide attempt & \\
\cline { 2 - 5 } & OR $(95 \% \mathrm{Cl})$ & $p$ value & OR $(95 \% \mathrm{Cl})$ & $p$ value \\
\hline Depression & $8.50(7.30,9.91)$ & $<0.001$ & $6.59(5.27,8.24)$ & $<0.001$ \\
\hline Anxiety & $4.19(3.69,4.77)$ & $<0.001$ & $4.08(3.15,5.35)$ & $<0.001$ \\
\hline Poor sleep quality & $2.99(2.49,3.62)$ & $<0.001$ & $3.38(2.28,5.23)$ & $<0.001$ \\
\hline CM & $2.00(1.78,2.25)$ & $<0.001$ & $2.64(2.14,3.26)$ & $<0.001$ \\
\hline TVD & $1.92(1.71,2.15)$ & $<0.001$ & $2.23(1.80,2.75)$ & $<0.001$ \\
\hline MOH & $1.79(1.56,2.05)$ & $<0.001$ & $2.95(2.35,3.68)$ & $<0.001$ \\
\hline MPS & $1.17(1.13,1.22)$ & $<0.001$ & $1.20(1.12,1.29)$ & $<0.001$ \\
\hline HIT-6 & $1.07(1.05,1.08)$ & $<0.001$ & $1.08(1.06,1.11)$ & $<0.001$ \\
\hline Headache days/month & $1.04(1.03,1.04)$ & $<0.001$ & $1.05(1.04,1.06)$ & $<0.001$ \\
\hline Married & $0.75(0.67,0.83)$ & $<0.001$ & $0.88(0.71,1.09)$ & 0.24 \\
\hline
\end{tabular}

Abbreviations: OR, odds ratio; $\mathrm{Cl}$, confidence interval; TVD, transient visual disturbance; MPS, Migraine Photophobia Score; HIT-6, six-item Headache Impact Test.

Table 4. Different models for the association of suicide risk with TVD and MPS

\begin{tabular}{|lllll|}
\hline & Suicidal ideation & & \multicolumn{2}{l|}{ Suicide attempt } \\
\cline { 2 - 5 } & OR $(95 \% \mathrm{Cl})$ & $\boldsymbol{p}$ value & OR $(95 \% \mathrm{Cl})$ & $\boldsymbol{p}$ value \\
\hline TVD+ Demographics & $1.86(1.66,2.09)$ & $<0.001$ & $2.17(1.75,2.69)$ & $<0.001$ \\
\hline TVD+ Demographics +Confounders & $1.44(1.19,1.73)$ & $<0.001$ & $2.18(1.53,3.14)$ & $<0.001$ \\
\hline MPS+ Demographics & $1.16(1.11,1.20)$ & $<0.001$ & $1.19(1.11,1.18)$ & $<0.001$ \\
\hline MPS+ Demographics+ Confounders & $1.08(1.03,1.13)$ & 0.001 & $1.07(0.98,1.16)$ & 0.1155 \\
\hline
\end{tabular}

Abbreviations: OR, odds ratio; $\mathrm{Cl}$, confidence interval; TVD, transient visual disturbance; MPS, Migraine Photophobia Score; HIT-6, six-item Headache Impact Test.

Demographics = age, gender, marital status; Confounders = depression, anxiety, poor sleep quality, HIT-6 score, headache frequency (days/ month), chronic migraine, medication overuse headache 
Table 5. Proposed operational criteria to characterize these migraine-associated transient visual disturbance

A. Transient visual disturbance not fulfilling 1.2.1 migraine with typical aura;

B. At least two of the following three characteristics:

1. Transient visual disturbance develops or spreads in $<5$ mins.

2. Transient visual disturbance lasts $<5$ minutes.

3. Transient visual disturbance occurs during headache phase.

\section{Figures}




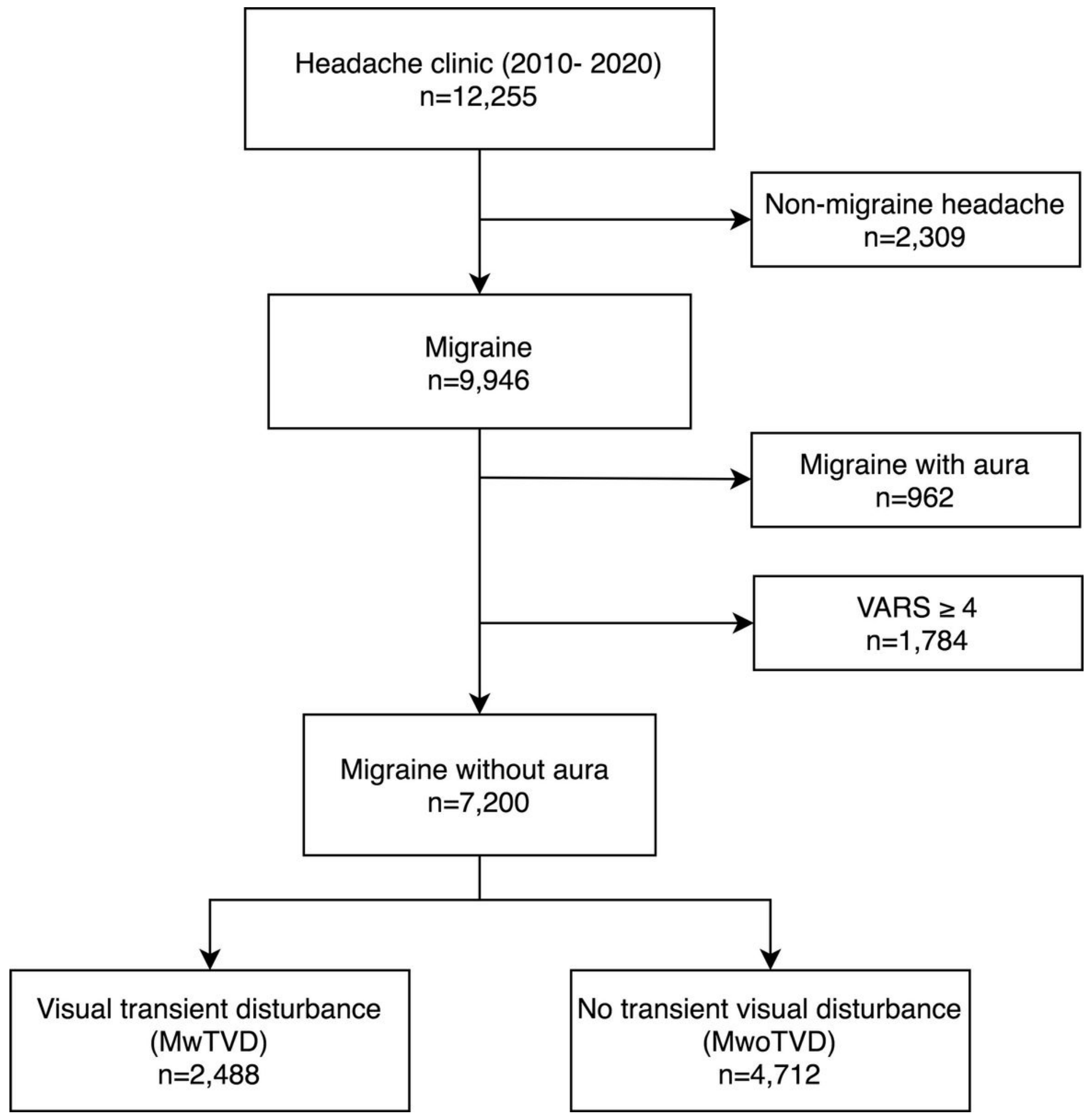

Figure 1

A flow chart of the patient enrollment process 


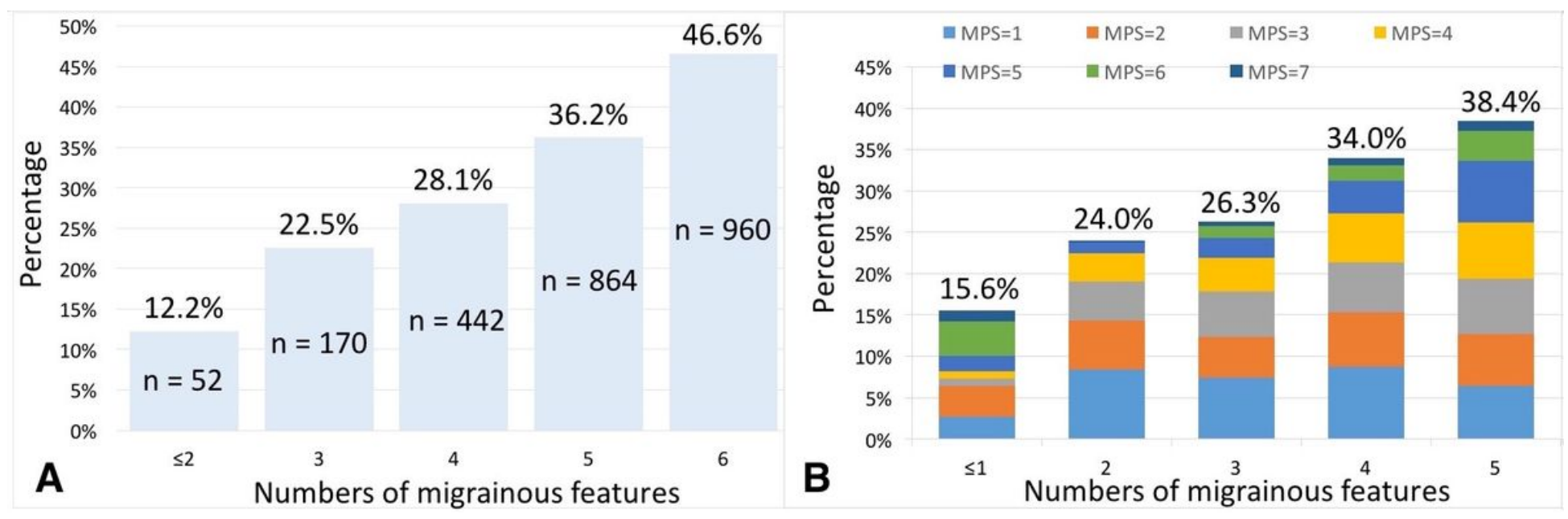

Figure 2

There is a trend for increasing TVD frequency with increasing migrainous features (Fig. 2A). While the TVD was reported in only $12.2 \%$ of $\mathrm{MO}$ patients with least migrainous features, its prevalence increased to $46.6 \%$ in those with all six migrainous features. To investigate whether patients with TVD were also more photophobic, we further compared the MPS between MWTVD and MwoTVD. Consistent with our speculation, MwTVD patients had higher MPS while comparing with MwoTVD (2.9 \pm 2.0 vs. $1.6 \pm 1.9, \mathrm{p}<$ 0.001 , Table 1). Moreover, we found MPS was a predictor of TVD (unadjusted OR $=1.37,95 \% \mathrm{Cl}=1.32-$ $1.42, \mathrm{p}<0.001$ ), and the result remained after gender and age were adjusted (adjusted $\mathrm{OR}=1.36,95 \% \mathrm{Cl}$ $=1.31-1.41, p<0.001)$. Similar to TVD, there is also a trend for increasing photophobia frequency with increasing migrainous features, expanding from $15.6 \%$ to $38.4 \%$ (Fig. 2B). 